\title{
Electroproduction and photoproduction of vector mesons and generalized vector-meson dominance
}

\author{
H. Fraas \\ Physikalisches Institut der Universität Würzburg, Würzburg, West Germany
}

M. Kuroda*

Deutsches Elektronen-Synchrotron DESY, Hamburg, West Germany

(Received 28 April 1977)

\begin{abstract}
Using generalized vector-meson dominance, electroproduction and photoproduction of vector mesons is studied. The unnatural-parity-exchange part of $\omega^{\prime}(1.2)$ production is estimated to be about $1 / 4$ of that of $\omega$ production. The off-diagonal transition model suggests the suppression of diffractive $\rho^{\prime}(1.2)$ and $\omega^{\prime}(1.2)$ production.
\end{abstract}

\section{INTRODUCTION}

Since the publication of the new data on the decay width $\Gamma(\rho \rightarrow \pi \gamma),{ }^{1}$ there has been much debate on $\mathrm{SU}(3)$-symmetry violation for radiative decays of vector mesons. ${ }^{2}$ In fact, the analyses of $\rho$ $\rightarrow \pi \gamma, \omega \rightarrow \pi \gamma$, and $\phi \rightarrow \pi \gamma$ decay rates suggest substantial violation of SU(3) symmetry for the $V P \gamma$ vertex.

As a possible solution of this problem, one of the present authors proposed ${ }^{3}$ on the basis of generalized vector-meson dominance (GVMD), a simple model of $\pi^{0}$ coupling to the two virtual photons. The radiative decay widths of vector mesons predicted in this model are in good agreement with the new data. One of the characteristics of the model is that it required the existence of $\omega^{\prime}$ around $1.2 \mathrm{GeV}$ with the width of the order of $400 \mathrm{MeV}$. There is experimental evidence ${ }^{4}$ on the existence of $\rho^{\prime}(1.2)$ but there is so far almost no evidence for $\omega^{\prime}(1.2)$. This may be attributed to the broadness of the $\omega^{\prime}$.
On the other hand, electroproduction and photoproduction of vector mesons give us, through the one-pion-exchange (OPE) amplitude, information on the $P V \gamma$ coupling constants, which are just the coupling constants that appear in radiative decays of vector mesons, and on the off-shell dependence of the virtual-photon form factors. Therefore, these reactions can be used in discussing $\mathrm{SU}(3)$ symmetry violation of $P V \gamma$ couplings, which is indicated in the radiative decay of vector mesons. In this paper, we focus our attention on unnaturalparity exchange, specifically the OPE part of these reactions, and compare the GVMD result with the naive VMD prediction. Our calculation shows that for $\omega$ production, a sizable deviation from the $\mathrm{SU}(3)$ symmetry can be expected from GVMD. Finally, using the "off-diagonal" transition model ${ }^{5}$ for the natural-parity-exchange part, we estimate $\rho^{\prime}$ and $\omega^{\prime}$ production cross sections.

Based on the GVMD model of Ref. 3 , the $\pi^{\circ}$ coupling to the two off-shell photons is expressed effectively as

$$
\begin{aligned}
\left\langle\pi^{0} \mid \gamma\left(p^{2}\right) \gamma\left(q^{2}\right)\right\rangle=e^{2} g \epsilon^{\alpha \beta \gamma \sigma} \epsilon_{\alpha}(p) p_{\beta} \epsilon_{\gamma}(q) q_{5} & {\left[\left(\frac{g_{\rho}}{f_{\rho}} \frac{m_{\rho}{ }^{2}}{m_{\rho}{ }^{2}-q^{2}}+\frac{g_{\rho^{\prime}}}{f_{\rho^{\prime}}} \frac{m_{\rho^{\circ}}{ }^{2}}{m_{\rho^{\prime}}{ }^{2}-q^{2}}+\cdots\right)\right.} \\
& \left.\times\left(\frac{g_{\omega}}{f_{\omega}} \frac{m_{\omega}{ }^{2}}{m_{\omega}{ }^{2}-p^{2}}+\frac{g_{\omega^{\prime}}}{f_{\omega}} \frac{m_{\omega}{ }^{2}}{m_{\omega}{ }^{2}-p^{2}}+\cdots\right)+(p-q)\right] .
\end{aligned}
$$

The coupling constants are normalized such that

$$
\frac{g_{V}}{f_{V}}+\frac{g_{V^{\prime}}}{f_{V^{\prime}}}+\cdots=1, \quad V=\rho \text { and } \omega
$$

The naive VMD prediction is the special case of Eq. (1), in which we use only the parent $\rho$ and $\omega$ and neglect daughter states. From the decay width of $\rho \rightarrow 2 \pi$ and $\omega \rightarrow 3 \pi$ we have $g_{\rho}{ }^{2} / 4 \pi=2.88$ \pm 0.9 and $g g_{\omega} g_{\rho}=16.5 \pm 0.8 \mathrm{GeV}$.
The dimensionless coupling constant $f_{\boldsymbol{V}}$ is determined by the leptonic decay of vector mesons, e.g., $f_{\rho}^{2} / 4 \pi=2.1 \pm 0.4$ and $f_{\omega}^{2} / 4 \pi=18.3 \pm 4.1$. Assuming the scaling law, ${ }^{6}$ we evaluate $f_{V_{n}}$ :

$$
\frac{f_{V_{n}}{ }^{2}}{f_{v}{ }^{2}}=\frac{m_{v_{n}}{ }^{2}}{m_{v}{ }^{2}},
$$

where $m_{V_{n}}^{2}=m_{V}^{2}(1+\lambda n)$ with $\lambda m_{V}^{2}=1 \mathrm{GeV}^{2}$. In subsequent arguments we use as the first approxi- 
TABLE I. Various coupling constants in our model. In the last column we give the values derived from $\Gamma(\omega \rightarrow \pi \gamma)$ and $\mathrm{SU}(3)$.

\begin{tabular}{lrrrcc}
\hline \hline$V$ & $f_{V}{ }^{2} / 4 \pi$ & $g_{V}{ }^{2} / 4 \pi$ & $f_{V}{ }^{2} / 4 \pi$ & $g_{V}{ }^{2} / 4 \pi$ & $\begin{array}{c}\text { SU(3) limit } \\
g_{V}{ }^{2} / 4 \pi\end{array}$ \\
\hline$\omega$ & 18.3 & 49.6 & 46.7 & 19.5 & 18.3 \\
$\rho$ & 2.1 & 2.88 & 5.5 & 0.16 & 2.1 \\
\hline \hline
\end{tabular}

mation up to the first daughter state $(n=1)$ of each vector-meson series. In fact, the analyses of the nucleon form factors indicate that there is a large contribution to the isoscalar nucleon form factor from the object of mass around $1.2 \mathrm{GeV},{ }^{7}$ which we identify as $\omega^{\prime}(1.2)$, the first Veneziano daughter of $\omega$. The author of Ref. 7 also observed that the contribution of $\omega$ and $\omega^{\prime}(1.2)$ cancel at large $q^{2}$, indicating

$$
m_{\omega}^{2} g_{\omega} / f_{\omega}+m_{\omega \cdot}^{2} g_{\omega \cdot} / f_{\omega} \sim 0 \text {. }
$$

In the isovector form factor, on the other hand, the contribution of $\rho^{\prime}(1.2)$, which corresponds to $\omega^{\prime}(1.2)$, turns out to be very small, which is quite consistent with our estimation

$$
\frac{g_{\rho^{\prime}}}{f_{\rho^{\circ}}} \cong 1-\frac{g_{\rho}}{f_{\rho}}=-0.17 \text {. }
$$

These properties of the form factors suggest that in the VMD calculation we should include the effect of at least the first daughter state.

From Eqs. (2)-(5) we obtain

$$
\begin{aligned}
& g_{\omega}=\frac{m_{\omega}{ }^{2}}{m_{\omega}{ }^{2}-m_{\omega}{ }^{2}} f_{\omega}, \\
& g_{\omega}=\frac{-m_{\omega} m_{\omega}}{m_{\omega}{ }^{2}-m_{\omega}{ }^{2}} f_{\omega} .
\end{aligned}
$$

The numerical values of various coupling constants are given in Table I, where we also give values for $g_{V}$ in the naive VMD model. From this table, one obtains the resultant $P V \gamma$ coupling constants as

$$
\begin{aligned}
& g_{\gamma \rho \boldsymbol{\Gamma}}=e g g_{\rho}=0.20 \mathrm{GeV}^{-1}, \\
& g_{\gamma \omega \boldsymbol{\Gamma}}=e g g_{\omega}=0.83 \mathrm{GeV}^{-1}, \\
& g_{\gamma \rho^{\prime \boldsymbol{r}}}=e g g_{\rho^{\prime}}=0.047 \mathrm{GeV}^{-1} \\
& g_{\gamma \omega^{\prime \boldsymbol{r}}}=e g g_{\omega^{\prime}}=0.52 \mathrm{GeV}^{-1} .
\end{aligned}
$$

Note that the width $\Gamma(\omega \rightarrow \pi \gamma)=880 \mathrm{keV}$ and $\mathrm{SU}(3)$ symmetry gives

$$
g_{\gamma \rho \pi}=0.26 \mathrm{GeV}^{-1}, \quad g_{\gamma \omega \boldsymbol{r}}=0.78 \mathrm{GeV}^{-1} .
$$

\section{PHOTOPRODUCTION OF VECTOR MESONS}

Let us first discuss photoproduction of vector mesons. ${ }^{8}$ Denoting the natural-and the unnatural- parity-exchange part of the production cross section of $\gamma p \rightarrow V p$ as $\sigma_{V}^{N}$ and $\sigma_{V}^{U}$, respectively, we can summarize the experimental results as follows. ${ }^{9}$ (1) $\sigma_{\rho}^{U}$ is negligibly small compared to $\sigma_{\rho}^{N} .{ }^{10}$ (2) $\sigma_{\omega}^{U}$ is, on the other hand, substantial at low energy: typically $\sigma_{\omega}^{U} \sim \sigma_{\omega}^{N}$ around $E_{\gamma} \sim 3 \mathrm{GeV}$, but at high energy $\sigma_{\omega}^{U}$ decreases and $\sigma_{\omega}^{N}$ is by one order of magnitude bigger than $\sigma_{\omega}^{U}$ at $E_{\gamma} \sim 9.3 \mathrm{GeV}$. (3) $\sigma_{\omega}^{N}$ is well parametrized by diffraction

$$
\sigma_{\omega}^{N}=c\left(1+D / E_{\gamma}\right) e^{A t}
$$

with $c=9.3 \pm 1.7 \mu \mathrm{b} / \mathrm{GeV}^{2}, D=1.3 \pm 1.2$, and $A=6.7$ $\pm 0.6 \mathrm{GeV}^{-2}$, while (4) $\sigma_{\omega}^{U}$ is well parametrized by OPE, where $g_{\gamma \omega \pi}$ is deduced from $\Gamma(\omega \rightarrow \pi \gamma)=880$ $\mathrm{keV}$.

Naive VMD predicts

$$
\frac{\sigma_{\rho}^{U}}{\sigma_{\rho}^{N}} \sim \frac{1}{81} \frac{\sigma_{\omega}^{U}}{\sigma_{\omega}^{N}}
$$

if $\sigma_{V}^{U}$ is saturated by OPE and $\sigma^{N}(\rho p \rightarrow \rho p)$ $=\sigma^{N}(\omega p \rightarrow \omega p)$. OPE is a good approximation insofar as the peripheral region is concerned and we assume, throughout this paper, the unnaturalparity exchange is saturated by OPE. The latter equality is quite natural if we consider the Pomeron exchange at high energy. Equation (9) explains why $\sigma_{\rho}^{U}$ is so small. In our GVMD calculations, the value $g_{\gamma \omega_{\boldsymbol{r}}}=0.83 \mathrm{is}$, within several percent, the same as the $g_{\gamma \omega \pi}=0.78$ predicted from $\Gamma(\omega \rightarrow \pi \gamma)$ $=880 \mathrm{keV}$. [cf. Eqs. (7) and (8)]. Therefore we cannot distinguish the two models by comparing $\sigma_{\omega}^{U}$ with experiment. Both models reproduce $\sigma_{\omega}^{U}$ successfully. On the other hand, $g_{\gamma o r}$ is by a factor of 1.3 smaller in our GVMD calculation than $g_{\gamma \rho \pi}$ given by $\mathrm{SU}(3)$ and the $\Gamma(\omega \rightarrow \pi \gamma)=880 \mathrm{keV}$. We can expect that $\sigma_{\rho}^{U}$ is by a factor $\sim 1.7$ smaller than in the naive VMD calculation. But as is mentioned above, $\sigma_{\rho}^{U}$ itself is so small that it is extremely difficult to detect the effect of this factor 1.7. Nevertheless, if it turns out that $\sigma_{o}^{U}$ and $\sigma_{\omega}^{U}$ do not obey SU(3), this would provide more evidence for SU(3) breaking in the $V P \gamma$ coupling. The conclusion is, of course, subject to the assumption of OPE saturation of $\sigma_{V}^{U}$.

\section{ELECTROPRODUCTION OF VECTOR MESONS}

In the case of electroproduction ${ }^{11,12}$ there is an additional contribution from the longitudinal photon. General formulas for OPE are given by Fraas $^{13}$ and summarized in the Appendix of Ref. 14. We consider here only $\omega$ electroproduction, because $\rho$ production is roughly saturated by the natural-parity exchange and gives no information on $P V \gamma$ couplings. Indeed, recent experimental data give the upperbound ${ }^{15}$

$$
\sigma^{U}\left(\gamma_{V} p \rightarrow \rho p\right) \leq(0.17 \pm 0.06) \sigma_{\text {tot }}\left(\gamma_{V} p \rightarrow \rho p\right) .
$$




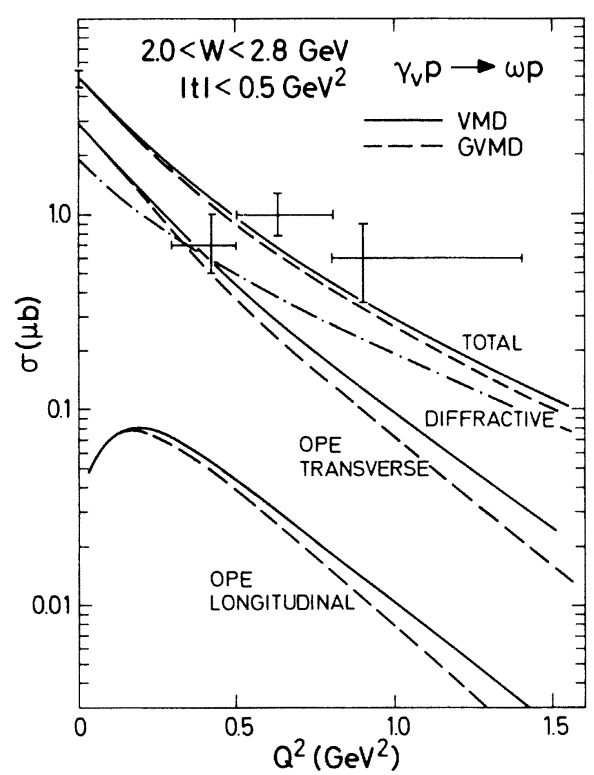

FIG. 1. $Q^{2}$ dependence of $\sigma\left(\gamma_{V} p \rightarrow \omega p\right)$ as predicted by the naive VMD model (solid curves) and by the GVMD model (dashed curves) for $2.0<W<2.8 \mathrm{GeV}$ and $|t|<0.5 \mathrm{GeV}^{2}$. The experimental data are from Ref. 14. The dasheddotted line is the diffractive cross section $\sigma^{N}\left(\gamma_{V} p \rightarrow \omega p\right)$, common to both models.

In the OPE term of $\omega$ electroproduction we use, as in Ref. 14, the Benecke-Dürr form factors, ${ }^{16}$ and the diffraction term is derived from photoproduction. The results are shown in Fig. 1 together with the experimental data. ${ }^{14}$ The solid line corresponds to the naive VMD model and the dashed line corresponds to our GVMD model. Our GVMD model predicts a smaller OPE cross section, e.g., at $Q^{2}=1.0 \mathrm{GeV}^{2}$; the ratio of the two curves for the OPE part is $\sim 1.4$. However, as the diffractive cross section dominates at large $Q^{2}$, the difference of the two models is smeared out in $\sigma_{\text {tot }}=\sigma^{U}+\sigma^{N}$ (see Fig. 1). In this respect an experiment which separates $\sigma^{U}$ from $\sigma^{N}$ is desirable. At the present stage, where we have no such experimental data, it is not possible to check the model. Again, if the OPE cross section is substantially smaller than the naive VMD model, especially at not-too-small $Q^{2}$, it will be an indication of SU(3)-symmetry breaking. We would like to stress the importance of separating $\sigma^{U}$ and $\sigma^{N}$ in comparing various models.

\section{IV. $\omega^{\prime}$ AND $\rho^{\prime}$ PRODUCTION}

Finally we estimate the cross sections $\sigma\left(\gamma p \rightarrow \omega^{\prime} p\right)$ and $\sigma\left(\gamma p \rightarrow \rho^{\prime} p\right)$ predicted in our GVMD model. For this purpose one needs several additional assumptions for the natural-parity-exchange amplitudes in both reactions. We follow the GVMD model with off-diagonal transitions of Ref. 5. This model includes contributions from a series of vector mesons $V_{N}$ and introduces, in addition to the elastic amplitudes $V_{N} p \rightarrow V_{N} p$, the off-diagonal transitions $V_{N} p \rightarrow V_{M} p$, i.e., diffraction dissociation of vector mesons. For the motivation of this procedure as well as for all details we refer to Ref. $5 .{ }^{17}$ Here we want only to list the main assumptions. These are the following:

(1) We assume the same mass spectrum of vector mesons as above, $M_{V_{N}}{ }^{2}=M_{V_{0}}{ }^{2}(1+\lambda N)$, and the same mass dependence, Eq. (3), for the photonvector-meson couplings $f_{\mathbf{V}_{N}}$ with alternating sign.

(2) We assume that the elastic amplitudes ${ }^{18}$ $T\left(V_{N} p \rightarrow V_{N} p\right)=T\left(V_{0} p \rightarrow V_{0} p\right)$ are the same for all vectors mesons $V_{N}$ (independent of the vector-meson mass).

(3) For the transition between neighboring vector mesons $V_{N}$ and $V_{N+1}$ we assume

$$
\frac{T\left(V_{N} p \rightarrow V_{N+1} p\right)}{T\left(V_{N} p \rightarrow V_{N} p\right)}=C_{N},
$$

where $C_{N}$ is a function of the masses $M_{N}$ and $M_{N+1}$

(4) For the mass dependence of diffraction dissociation we take a simple power law

$$
\begin{aligned}
& \frac{T\left(V_{M} p \rightarrow V_{N} p\right)}{T\left(V_{M} p \rightarrow V_{M+1} p\right)}=\left(\frac{m_{M}}{m_{N}}\right)^{2 p+1} \\
& \quad \text { for } N \geq M+2, M \geq 0 .
\end{aligned}
$$

Here we fix the parameters $p$ and $C_{N}$ to be $p=\frac{1}{2}$ and $C_{N}=\left(m_{0} / m_{1}\right)^{2}=\frac{1}{3}$ (independent of $\left.N\right)$. The general feature of these assumptions is that the dominant contribution to the hadronic diffractive $\rho^{\prime}\left(\omega^{\prime}\right)$ and $\rho^{\prime \prime}\left(\omega^{\prime \prime}\right)$ production amplitudes $T$ comes from the diagonal part and that as the off-diagonality increases the contribution decreases.

Then after a simple calculation we find that the natural-parity-exchange cross section for photoproduction of $\rho^{\prime}$ or $\omega^{\prime}$ is strongly suppressed:

$$
\sigma^{N}\left(\gamma p \rightarrow\left\{\begin{array}{l}
\rho^{\prime} \\
\omega^{\prime}
\end{array}\right\} p\right) \sim\left(\frac{1}{20}-\frac{1}{100}\right) \sigma^{N}\left(\gamma p \rightarrow\left\{\begin{array}{l}
\rho \\
\omega
\end{array}\right\} p\right) .
$$

This is clearly a consequence of including diffractive dissociation of vector mesons and of the alternative-sign assumption for the $\gamma-V_{N}$ couplings. Of course, the numerical value of the suppression factor in the above equation cannot be taken too seriously. The qualitative result of the strong suppression of diffractive $\omega^{\prime}$ and $\rho^{\prime}$ photoproduction does, however, not depend crucially on the detailed assumptions (i.e., the values of $C_{N}$ and $p$ ) of the model discussed. This would explain why it is so hard at high energy to produce $\rho^{\prime}$ and $\omega^{\prime}$. In contrast, $\rho^{\prime \prime}(1600)$ and $\omega^{\prime \prime}(1600)$ production is estimated in this model as 


$$
\sigma^{N}\left(\gamma p \rightarrow\left\{\begin{array}{l}
\rho^{\prime \prime} \\
\omega^{\prime \prime}
\end{array}\right\} p\right) \sim \frac{1}{6} \sigma^{N}\left(\gamma p \rightarrow\left\{\begin{array}{l}
\rho \\
\omega
\end{array}\right\} p\right)
$$

that is, we can expect a sizable diffractive production of $\rho^{\prime \prime}$ and $\omega^{\prime \prime}$.

Note that in photoproduction experiments, $\gamma p$ $\rightarrow p+M M$ and $\gamma p \rightarrow p \pi^{+} \pi^{-}+M M$, a resonance state of mass $1250 \mathrm{MeV}$ has been seen. ${ }^{19}$ The production cross section is as big as $1-2 \mu \mathrm{b}$ and the dominant decay mode is into $\omega \pi^{0}$. The spin and parity of this state is either $J^{P}=1^{+}$or $1^{-}$, corresponding to $B$ or $\rho^{\prime}$, and both assignments are compatible with the data. In view of the smallness of $\sigma^{N}\left(\gamma p \rightarrow \rho^{\prime} p\right)$ predicted in our model, we regard this object as a $J^{P}=1^{+} B$ meson and we do not identiy it with the object of mass $1250 \mathrm{MeV}$, which is found as an enhancement in the $e^{+} e^{-} \rightarrow \omega \pi^{0}$ reaction. The latter is regarded as $\rho^{\prime}(1.2)$ (see Leith in Ref. 4). It is therefore crucial in the off-diagonal transition model that the former object is not $\rho^{\prime}(1.2)$.

As to the OPE part of $\omega^{\prime}$ and $\rho^{\prime}$ one notices from Table I that $\left(g_{\rho_{\rho}} / g_{\rho}\right)^{2} \sim 0.06$, whereas $\left(g_{\omega} / g_{\omega}\right)^{2} \sim 0.4$. Therefore $\sigma_{\rho^{\prime}}^{U}\left(\right.$ and $\left.\sigma_{\rho^{\circ}}\right)$ is expected to be quite small. For $\omega^{\prime}$ production, however, one has considerable contributions from OPE, so that at not-too-high energies it should be possible to see $\omega^{\prime}$. Expected $\omega^{\prime}$ production cross sections are shown in Fig. 2. The dominant decay mode of $\omega^{\prime}$ is $\omega^{\prime} \rightarrow \rho \pi$ (or $\left.3 \pi\right){ }^{3}$

The results do not change much for $Q^{2} \neq 0$ so that, in electroproduction, the diffractive contribu-

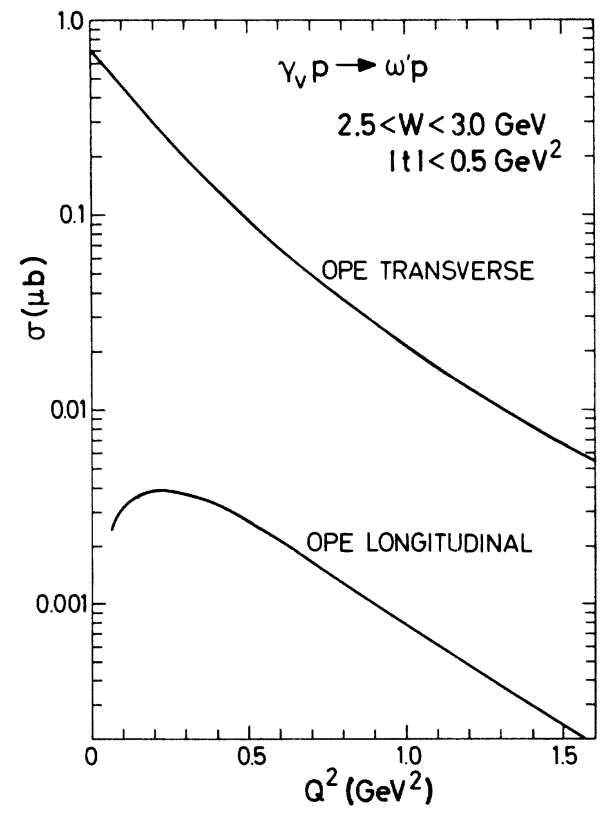

FIG. 2. $Q^{2}$ dependence of $\sigma\left(\gamma_{V} p \rightarrow \omega^{\prime} p\right)$ as predicted by the GVMD model of Ref. 3 for $2.5<W<3.0 \mathrm{GeV}$ and $|t|<0.5 \mathrm{GeV}^{2}$. tion is small and the OPE part of the $\omega^{\prime}$ production cross section is about $\frac{1}{3}$ of the OPE part of the $\omega$ production cross section.

\section{COMMENTS}

Finally several comments are in order.

(1) One obtains the ratio $\sigma_{\omega} / \sigma_{\rho}$ at $Q^{2}=0$ from the photoproduction experiment

$$
\frac{\sigma_{\omega}}{\sigma_{\rho}} \sim \frac{\sigma_{\omega}^{N}}{\sigma_{\rho}^{N}}\left(1+\frac{\sigma_{\omega}^{U}}{\sigma_{\omega}^{N}}\right)=0.27 .
$$

This ratio decreases as $Q^{2}$ increases in both GVMD and naive VMD models since, as is seen from Fig. 1, $\sigma_{\omega}^{U} / \sigma_{\omega}^{N}$ is a decreasing function of $Q^{2}$. Experimental data show a flat ${ }^{14}$ or rather a slightly increasing behavior of $\sigma_{\omega} / \sigma_{\rho}$ with increasing energy. Possibly this discrepancy could for the peripheral region be due to the Benecke-Dür form factors we used. By these form factors a rather strong additional $Q^{2}$ dependence is introduced which with increasing $Q^{2}$ damps the OPE cross section.

(2) If the narrow vector resonance recently discovere $\mathrm{d}^{20}$ around $1100 \mathrm{MeV}$ is isoscalar, we can alternatively use it in place of $\omega^{\prime}(1.2)$, because what is essential in our GVMD model is the existence of an isoscalar object around $1.2 \mathrm{GeV}$, which contributes strongly to the isoscalar form factor. Without further information we cannot go into detail at this point.

(3) An analytic model of pion coupling to the two virtual photons is proposed in terms of the hypergeometric function. ${ }^{21}$ This model is completely within the framework of SU(3) symmetry and cannot explain the small width $\Gamma(\rho \rightarrow \pi \gamma)$. Nevertheless it is interesting to compare the model with ours. Pulling out $g_{\gamma \omega^{\prime}, \mathrm{s}}$ and $g_{\gamma \rho^{\prime} \mathrm{r}}$ in both models and comparing $g_{\gamma \omega^{\prime}, \mathbf{s}} / g_{\gamma \omega \boldsymbol{r}}$ and $g_{\gamma \rho^{\prime} \boldsymbol{r}} / g_{\gamma \rho \pi}$, we find that our coupling is reproduced by setting $\gamma=2$ for the $\omega$ series and $\gamma=1$ for the $\rho$ series, while $\gamma=\frac{3}{2}$ in the or iginal model. Here, $\gamma$ is a fixed parameter corresponding to quark-pseudoscalar-meson trajectories appearing in Ref. 21. The fact that we need two different values of $\gamma$ for the $\omega$ series and the $\rho$ series is merely the reflection of the $\mathrm{SU}(3)$ breaking nature of our GVMD model. For these values of $\gamma$,

$$
\begin{aligned}
& \qquad g_{\gamma \omega \boldsymbol{r}}=g_{\rho \omega \boldsymbol{r}} \frac{1}{3 m_{\omega}^{2} \alpha^{\prime} f_{\rho}} \\
& \text { and } \\
& \qquad g_{\gamma \rho \boldsymbol{r}}=g_{\rho \omega \boldsymbol{r}} \frac{1}{2 m_{\rho}^{2} \alpha^{\prime} f_{\omega}},
\end{aligned}
$$

which indicates that $\mathrm{SU}(3)$ is broken by a factor $g_{\text {rwr }} / 3 g_{\text {ror }}=\frac{2}{3}$ but in an opposite direction to what the experimental data show. 
(4) We have shown in this paper that our GVMD model is consistent with the present experimental data on $\omega$ and $\rho$ photoproduction and electroproduction and that to distinguish various models a very accurate experiment which separates naturaland unnatural-parity exchange is required. We have also shown that the $\rho^{\prime}$ and $\omega^{\prime}$ diffractive production would be quite small, while $\rho^{\prime \prime}$ and $\omega^{\prime \prime}$ diffractive production can be as big as $\frac{1}{6}$ of $\rho$ and $\omega$ production.

(5) The experiment which allows us to separate $\sigma^{U}$ from $\sigma^{N}$ is highly desirable in comparing the various models for OPE amplitude and $V P \gamma$ couplings. Experiments which definitely determine the spin and parity of the object at $1250 \mathrm{MeV}$ observed in photoproduction experiments would also be desirable.

\section{ACKNOWLEDGMENTS}

The authors would like to express their thanks to T. F. Walsh for valuable comments and reading of the manuscript. They also thank G. Wolf and H. Spitzer for providing experimental information. H. F. thanks Professor H. Joos for his kind hospitality at DESY, where part of the work was performed, and M. K. thanks the Alexander von Humboldt Foundation for financial support.
*Work supported by Alexander von Humboldt Foundation.

${ }^{1}$ B. Gobbi et al., Phys. Rev. Lett. 33, 1450 (1974); 37, 1439 (1976).

${ }^{2}$ For a review see, for example, R. G. Moorhouse, CERN Report No. CERN TH 2103, 1976 (unpublished). ${ }^{3}$ K. Fujikawa and M. Kuroda, DESY Report No. DESY 76/23 (unpublished); and Lett. Nuovo Cimento 18, 539 (1977).

${ }^{4}$ P. Frenkiel et al., Nucl. Phys. B47, 61 (1972); V. Alles-Borelli et al., Nuovo Cimento 30A , 136 (1975); M. Conversi et al., Phys. Lett. $\overline{52 B}, 493$ (1974); G. Cosme et al ., Orsay Report No. LAL 1287, 1976 (unpublished). See also D. Bellini et al., Lett. Nuovo Cimento 14, 418 (1975); Phys. Lett. 61B, 96 (1976). See also F. Close, in Storage Ring Physics, proceedings of the International Colloquium, Flaine Haute Savoie, edited by J. Trân Thanh Vân, 1976 (CNRS, Paris, 1976), p. 169.

D. W. G. S. Leith, in Electromagnetic Interactions of Hadrons, edited by A. Donnachie and G. Sahw (Plenum, New York, to be published).

${ }^{5}$ H. Fraas et al., Nucl. Phys. B86, 346 (1975); B B88, 301 (1975).

${ }^{6}$ K. Fujikawa, Phys. Rev. D 4, 2794 (1971); J. J. Sakurai and D. Schildknecht, Phys. Lett. 40B, 121 (1972); A. Bramon et al ., ibid 41 B, $609 \overline{(1972)}$.

${ }^{7}$ G. Höhler et al., Nucl. Phys. B114, 505 (1976); G. Höhler, in Proceedings of the International Summer Institute on Theoretical Particle Physics, edited by J. G. Körner et al. (Springer, Hamburg, 1975).

${ }^{8}$ For a review of photoproduction experiments, see A. Silverman, in Proceedings of the 1975 International Symposium on Lepton and Photon Interactions at High Energies, Stanford, California, edited by W. T. Kirk (SLAC, Stanford, 1976), p. 355.

${ }^{9} \mathrm{~J}$. Ballam et al., Phys. Rev. D 7, 3150 (1973).

${ }^{10}$ It is quoted in Ref. 8 that $\left|T_{1} / \bar{T}_{0}\right|^{2}=0.03 \pm 0.07$ at $E_{\gamma}$ $\sim 4.3 \mathrm{GeV}$, where the suffix denotes the isospin exchanged in the $t$ channel.

${ }^{11}$ For a review of electroproduction, see G. Wolf, in Proceedings of the 1975 International Symposium on Lepton and Photon Interactions at High Energies, Ref. 8.

${ }^{12}$ For a review of muoproduction, see R. F. Mozley, in Proceedings of the 1975 International symposium on Lepton and Photon Interactions at High Energies, Ref. 8.

${ }^{13}$ H. Fraas, Nucl. Phys. B36, 191 (1972); B49, 253 (1973).

${ }^{14}$ P. Joos et al., Nucl. Phys. B122, 365 (1977).

${ }^{15}$ P. Joos et al., Nucl. Phys. B113, 53 (1976).

${ }^{16} \mathrm{~J}$. Benecke and P. H. Dürr, Nuovo Cimento $56 \mathrm{~A}, 269$ (1968).

${ }^{17}$ The first part of this section is based on the discussion with B. J. Read and D. Schildknecht.

${ }^{18}$ Since we are discussing photoproduction near the forward direction we can restrict ourselves to the $s$ channel helicity amplitudes for transversely polarized photons and vector mesons.

${ }^{19}$ R. Anderson et al., Phys. Rev. D 1, 27 (1970);

J. Ballam et al., Nucl. Phys. B76, 375 (1974);

E. Rabe et al ., DESY Report No. DESY-FI-F1/2 (unpublished).

${ }^{20}$ S. Bartalucci et al., DESY Report No. 76/43 (unpublished); Nuovo Cimento 39A, 374 (1977).

${ }^{21}$ Etim-Etim and M. Greco, CERN Report No. CERN TH 2174, 1976 (unpublished). 